\title{
Teaching Astronomy at the University of South Africa
}

\author{
By W.F. Wargau ${ }^{1}$, B. Cunow ${ }^{1}$ AND C.J.H. Schutte ${ }^{2}$ \\ ${ }^{1}$ Department of Mathematics, Applied Mathematics and Astronomy, University of South \\ Africa, P. O. Box 392, Pretoria 0001, \\ ${ }^{2}$ Chief Executive Director: Science, Technology and Informatics, University of South Africa, \\ P. O. Box 392, Pretoria 0001,
}

\section{The development of distance education in South Africa: historical background and the University of South Africa}

The University of South Africa celebrates its 50th anniversary this year. Over this period it grew, becoming one of the largest tertiary distance education institutions and the largest university on the African continent.

South Africa always had a mixed racial population with each group having its own culture. This difference between people is further aggravated by differences in the level of "westernisation". Furthermore, South Africa also suffers from an extreme urbanisation problem where on the one hand we find modern cities and on the other tribal groups. All these factors led to a differentiation of the population into a first world and third world component.

In 1858 the government of the Cape Colony decided to institute a board of public examiners in literature and science. The task was to set syllabuses and to set and conduct examinations at college level. In 1864 this board instituted a certificate which was equivalent to the British matriculation certificate. The board only conducted examinations, but offered no training. In 1873 the parliament of the Cape of Good Hope decided to establish the University of the Cape of Good Hope. The University still was an examining body only, which set syllabi, conducted examinations and held graduation ceremonies. Its degrees were recognised by the British Commonwealth.

This institution had to face some very adverse criticism from those who felt that a university can only function in a direct teaching situation, that it was too "foreign"(British) for the country and that it was a mere factory of certificates.

In 1916 the Parliament of the newly created Union of South Africa decided to change the name of the university to "University of South Africa" (Unisa) which started operating in 1918. In the same year the university moved to Somerset House in Pretoria.

Parallel to the University of South Africa as an examining body for colleges etc., a number of residential universities were established over the years. A large number of students who did not want to receive their tuition at colleges or seminaries but were not able to study at residential universities wished to register as external students at Unisa. Finally, the student body grew so large that much discussion started around a concept of distance education at university level. In 1946 the University of South Africa decided to offer distance education. The first director, Prof. A.H.J. van der Walt, laid the ground for the present university. The total student numbers grew from 5500 in 1955 , over 40000 in 1975 to 128000 in 1995 . The percentages of black and coloured students were $21 \%$ in 1955 , dropped to $16 \%$ in 1975 and reached $51 \%$ in 1995 . In terms of gender, the present student body is made up of $46 \%$ male and $54 \%$ female students.

Since 1994 the role of Unisa as a distance education institution has become more important than before in order to provide adequate education for all population groups. The main aim of distance education in the new South Africa is to afford equal education 
and employment opportunities to all qualified persons within and beyond the borders of South Africa.

\section{The astronomy curriculum}

Historically the subject of astronomy has been taught in the Department of Mathematics and Applied Mathematics since 1960. During the first decade astronomy was offered at undergraduate level and was seen as a part of the degree in Mathematics or Applied Mathematics. In the 1970s an Honours course was developed which could be selected by the student and constituted part of the Mathematics/Applied Mathematics Honours. In 1986 astronomy formally became a subdepartment. Subsequently, a full curriculum including some practical components at undergraduate and postgraduate levels was developed.

The University of South Africa offers modules, papers and courses which run over a full year. The smallest study unit is the module which is equivalent to a 50 hour work unit. A paper is reckoned as two modules and a course as three or more modules. The Bachelor of Science degree comprises 30 modules, the Honours Bachelor of Science five papers, the Master of Science a selected project and the PhD own research.

As prerequisite for the study of astronomy, mathematics at matriculation level is required. The astronomy curriculum comprises modules at undergraduate levels and papers at Honours level, while on Master and $\mathrm{PhD}$ level selected topics including own research can be chosen.

Astronomy can be selected as the only major for the BSc degree or as a double major in the combinations astronomy and mathematics, astronomy and applied mathematics, astronomy and either physics or theoretical physics. For the double major we strongly recommend the combination astronomy and physics.

When Astronomy is selected as the only major, the following options are available:

- 19 compulsory modules

- 9 astronomy modules

- 6 mathematics modules

- 4 physics modules

- 11 remaining modules which can be selected from other BSc subjects, e.g., chemistry, computer science, statistics, geology, geography or biology. At least four modules must be on second-year and four on third-year level.

Astronomy as part of a astronomy/physics double major comprises:

- 27 compulsory modules

- 9 astronomy modules

- 6 mathematics modules

- 12 physics modules

- 3 remaining modules which can be selected in one or more of the other BSc subjects. We strongly recommend that the student enrolls for some chemistry modules.

Students who have registered for a double major can pursue both subjects at Honours level. In our example that would be physics and astronomy.

At Honours level we offer six papers of which the student must at least enrol for three while the other two can be chosen from physics, mathematics, applied mathematics, chemistry or computer science. Of the six astronomy papers, one is compulsory, namely "Stellar Structure and Evolution".

The tutorial material comprises a Study Guide and up to three Tutorial Letters which the student receives on enrolement for a module/paper. The Tutorial Letter contains general hints, assignments, guidelines for examination admission and procedures, recom- 
mendation for further reading; information on textbooks, and information about a video tape containing some comments on the curriculum, our facilities and where the lecturers introduce themselves. The video tape can be loaned from the Unisa Library.

Some Study Guides are wrap-around guides, while others are self-contained. A Study Guide contains all the material, such as explanations, tables and appendices which the student may need to solve the assignments. Contact with students is currently established via mail and telephone. Discussion classes are not offered on a regular basis because the number of students is too small. Lecturers invite students to the main campus in Pretoria: local students make regularl use of this, while distant students come only occasionally. For local students evening classes are arranged on an ad hoc basis. They usually cover selected topics from the undergraduate curriculum, but are not meant as regular lectures.

In the following we would like to look at the modules and papers more in detail. On first-year level we offer two modules. One is a general introduction and the other deals with spherical astronomy and Kepler orbits. For the introductory module the student numbers vary between 70 and 100 , while for the second one between 30 and 50 . The pass rate versus admission to examination is around $75 \%$. On second-year level, we offer three modules. The first one contains basic instrumentation including optics, telescopes, image formation, spectroscopy and photometry; the second one observational astrophysics including magnitude system, spectral and luminosity classification of stars, interstellar medium, our Galaxy and extragalactic objects; the third module is the introductory practical. Student numbers on this level vary between five and ten students per module. On third-year level we offer four modules. The following topics are covered: astrophysical principles including atomic structure, electromagnetic radiation and gas laws, ionization and excitation processes and black body radiation; radiation transport including line formation, analysis of stellar spectra and stellar structure equations; astrodynamical principles including stability of stellar systems, stellar motions and galaxy dynamics. In addition, we offer an advanced astronomy practical. The student numbers vary between two and five students per module.

At Honours level we cover the following topics. A paper on stellar structure and evolution which is compulsory; papers on interstellar medium, radio astronomy, observational techniques and cosmodynamics. In addition we offer a project paper where the student can choose between a theoretical and practical oriented project. "Theoretical" in this context means that the student reviews recent research on a certain topic, while "practical oriented"involves observations at South Africa's national observatory in Sutherland, at the radio astronomy observatory in Hartebeesthoek, or at the Unisa observatory. The aim is to familiarise the student with observing and reduction procedures. Student numbers are typically three per year.

\section{The Unisa Observatory}

Astronomy is a practical science. It is unimaginable that the entire curriculum is based only on the printed word. Although the situation has improved in the past years due to the advent of electronic media, there is no real substitute for face-to-face contact for practicals. In order to cater for practicals Unisa had set up obligatory practical courses for physics, chemistry, geology and the life sciences in the seventies.

Originally, the tuition for astronomy was entirely based on the printed word, and contact to students was only via telephone, or by occasional visits. As from 1986 the need for face-to-face contact became more and more pressing with the foundation of the Subdepartment of Astronomy and with the introduction of a fully-fledged curriculum including undergraduate and postgraduate levels. The idea of building a small but modern 
observatory which could be used for training students was conceived in 1987. Although the idea was very challenging, it would be very expensive. On the other hand, such a project was unique to South Africa and for the African continent. The University officials were persuaded to grant the money for building the observatory. On 17 August 1992 the Unisa Observatory was inaugurated by the then Principal, Prof. van Vuuren. In 1993 a practical module on introductory level was launched, which was followed by an advanced practical module in 1994 .

The technical equipment of the observatory comprises the following:

- A Compustar C14 computer-controlled 14-inch Schmidt-Cassegrain telescope.

- A fully motorized dome

- An automated PMT-based UBV photometer filters.

- A Daystar 0.6 Angstrom ATM filter and energy-rejection cover.

- Spath-crystal micrometer.

- A two $(600,1200$ grooves mm-1) grating spectroscope with reducer, reference source and photographic recording.

- A CCD imaging camera with UBVRI and clear filters.

- Audio-video and photographic equipment including reproduction facilities.

- A 586 Pentium $100 \mathrm{MHz}$ and a $486 \mathrm{DX} 33 \mathrm{MHz}$ PC.

- An excellent slide and video collection.

- Complete set of filmcopies of ESO/SERC $\mathbf{J}$ and $R$ surveys.

The observatory premises include a library, a lecture room, a kitchen, a restroom and sanitary facilities. In other words, the observatory is self-contained.

The introductory practical, which is part of the second year course, includes thorough training at the telescope, planning and preparation of astronomical observations, astrophotography and solar observations. The advanced practical is part of third year and gives a thorough training in professional observing techniques such as UBV photometry, spectroscopy and UBVRI CCD photometry. Both are compulsory and are scheduled for the South African winter month July, where cloudless conditions are fairly predictable for Pretoria. Each practical runs over two weeks. We offer no practical on first year level because the student numbers are relatively high and an adequate theoretical foundation is needed before a student can benefit from a concentrated practical session. The practicals render face-to-face contact possible for students - which is so important for the practical courses of a distance teaching university.

At Honours level, we offer a project paper which includes work at the observatory. Besides doing a literature study the student can prepare and carry out observations at the telescope. Presently, students are mainly involved in testing the equipment. At Master level the student may choose a dissertation which is linked entirely to the observatory. One of our Master students, for example, wrote a dissertation on the evaluation of the Unisa photometric system. Alternatively the student can do his/her project paper as well as his/her Master dissertation at the South African Astronomical Observatory in Cape Town/Sutherland or at the radio astronomy facilities at Hartebeesthoek. For overseas students special arrangements are made with local universities.

Allocation of observing time at international observatories becomes more and more difficult for graduates. Although they do not lack research ideas, they do not have the required know-how to handle sophisticated equipment. Observatory committees which schedule observing time are reluctant to allocate precious observing time to newcomers. This deficit can be partly ironed out by giving the students sufficient training at a modern but scaled-down observatory. The Unisa observatory is ideal for this kind of training. 


\section{Future developments}

\subsection{Collaboration}

A very recent project includes combining the study of Physics and Astronomy at Unisa (distance teaching) with that of the University of Pretoria (UP; residential). The idea is the following: first year astronomy is offered at UP; from the second year onwards the student may carry on with astronomy at Unisa, while the physics subjects are taught at UP. The student obtains a BSc degree in Physics from UP. It is planned to implement this scheme in 1997.

\subsection{Students on Line}

The communication system which Unisa has developed for the two-way flow of information between the university and the student can be described as dependent upon the printed word. The main carrier of information is the postal system. Over the past decades this has been enriched with modern technology, such as telephone, audio tapes and student centres at locations outside Pretoria. More recently computer-aided instruction and Picture'Tel interactive video classes were introduced.

As more and more students have access to electronic media, we thought of a more effective way for carrying out Honours and Masters projects in astronomy. At the observatory the student plans the observing run and obtains the data, which will be transferred to a file server and the student can retrieve them via the Internet. The data reduction and analysis can then be performed at home according to instructions supplied by the Lecturers.

In the light of Unisa's total student numbers the Department of Production yearly prints about 500 million pages of study material, the Department of Dispatch yearly handles millions of postal articles and the Department of Assignments annually handles millions of assignments. This was made feasible by implementing one of the best computer management systems in the world which was developed by Unisa. In recent years student numbers and therefore costs increased tremendously so that Unisa is now facing big problems. If more and better study material is to be printed, several million Rand are needed immediately. The handling of assignments which mainly depends on machines and human hands is also getting more and more expensive. A third problem is the slow speed of mail delivery in South Africa which causes severe delays in receiving and sending assignments.

A powerful solution to these problems is the use of the World Wide Web (Internet) service which is fairly easy accessible for those who have access to a PC. We can make excellent use of this source in the following way:

- communication between students and lecturers through e-mail

- the students can download study material and even parts of prescribed and recommended books

- students can submit assignments which are electronically registered and marked by lecturers without ever printing them out; they are then electronically returned to the students

- students can search through the catalogues of the Unisa library and order books and reprints electronically

- students can "talk" to other students through an electronic forum

- students can enrol at the University without having to write or to travel to a Unisa office

In order to achieve these aims, an electronic server at Unisa, called SOL (Students On Line) has been set up. Unisa is now in the process of implementing the server step by 
step. At the end of the day it will save the University an enormous amount of paper work. The idea is to develop new teaching methods and to redesign our study material for the new medium. It will take some time for the students and lecturers to get acquainted with the new system. But in the year 2000 and beyond the University will probably operate in a different way from that of today. There will always be made provision for students who prefer the paper model and/or who do not have the necessary computer access. 